\title{
Dysfunctional high-density lipoprotein and atherogenesis
}

\author{
Constantine E. Kosmas ${ }^{1}$, Delia Silverio², Andreas Sourlas ${ }^{3}$, Peter D. Montan², Eliscer Guzman ${ }^{1}$ \\ 'Department of Medicine, Division of Cardiology, Montefiore Medical Center, Bronx, NY 10467, USA. \\ ${ }^{2}$ Cardiology Clinic, Cardiology Unlimited PC, New York, NY 10033, USA. \\ ${ }^{3}$ School of Medicine, University of Crete, Heraklion 71003, Greece.
}

Correspondence to: Dr. Constantine E. Kosmas, Department of Medicine, Division of Cardiology, Montefiore Medical Center, 168-24 Powells Cove Blvd., Beechhurst, NY 11357, USA. E-mail: cekosmas1@gmail.com

How to cite this article: Kosmas CE, Silverio D, Sourlas A, Montan PD, Guzman E. Dysfunctional high-density lipoprotein and atherogenesis. Vesse/ Plus 2019;3:2. http://dx.doi.org/10.20517/2574-1209.2018.79

Received: 24 Nov 2018 First Decision: 22 Dec 2018 Revised: 23 Dec 2018 Accepted: 30 Dec 2018 Published: 21 Jan 2019

Science Editor: Alexander N. Orekhov Copy Editor: Cui Yu Production Editor: Huan-Liang Wu

\begin{abstract}
High-density lipoprotein (HDL) plays a major role in reverse cholesterol transport (RCT) but also exhibits, antiinflammatory, endothelial/vasodilatory, anti-thrombotic, antioxidant, anti-aggregating, anticoagulant and cytoprotective functions, which enhance its protective effect against cardiovascular disease. However, the function of HDL is dependent upon genetic, environmental and lifestyle factors. Modification of the protein or lipid components of HDL in certain conditions may convert the HDL particles from anti-inflammatory to pro-inflammatory and pro-atherogenic by limiting their ability to promote RCT and to prevent LDL modification. In our review, we will present the clinical and scientific data pertaining to the factors and conditions that impair HDL functionality and we will discuss the effects of dysfunctional HDL on atherogenesis.
\end{abstract}

Keywords: High-density lipoprotein, high-density lipoprotein functionality, atherogenesis, cardiovascular disease

\section{INTRODUCTION}

There is extensive clinical evidence showing that there is a clear inverse relationship between serum highdensity lipoprotein-cholesterol (HDL-C) concentrations and the risk for cardiovascular disease (CVD), which is independent of the concentration of low-density-lipoprotein cholesterol (LDL-C) ${ }^{[1]}$. Actually, in a large meta-analysis, which included 20 randomized controlled trials with 543,210 person-years of follow-up and 7,838 myocardial infarctions, it was shown that, after adjustment for on-treatment LDL-C levels, age, hypertension, diabetes, and tobacco use, statins do not affect the relationship between HDL-C concentration

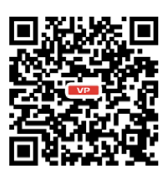


and cardiovascular risk, so that low HDL-C concentrations continue to exhibit a significant, independent association with increased CVD risk despite statin therapy ${ }^{[2]}$.

The cardioprotective effects of HDL are exerted mainly via its role in the reverse cholesterol transport (RCT) pathway, by promoting the removal of cholesterol from peripheral cells and thus inhibiting foam cell formation and preventing atherogenesis. Furthermore, HDL promotes endothelial repair, decreases the expression of endothelial adhesion molecules and possess anti-inflammatory, antioxidant, antiaggregatory and anticoagulant properties. Thus, it becomes evident that the cardioprotective effect of HDL goes beyond $\operatorname{RCT}^{[3,4]}$.

On the other hand, there is ample clinical evidence showing that HDL functionality, more than HDL-C concentration per se, plays a crucial role in atheroprotection ${ }^{[5,6]}$. HDL functionality is assessed by the cholesterol efflux capacity (CEC), which determines the ability of HDL to accept cholesterol from macrophages for excretion into the liver. CEC has been shown to be an excellent predictor of atherosclerotic disease $^{[7]}$.

Furthermore, it is known that under certain conditions, such as the oxidative environment of the acute-phase response, the HDL particles may lose their anti-inflammatory properties and become proinflammatory ${ }^{[8]}$.

In our review, we will present the clinical and scientific data pertaining to the factors and conditions that impair HDL functionality and we will discuss the effects of dysfunctional HDL on atherogenesis.

\section{HDL STRUCTURE AND HETEROGENEITY}

HDL is synthesized in the intestine and the liver and consists of a heterogeneous group of particles, which differ in density, size, electrophoretic mobility, and apolipoprotein content ${ }^{[5,9]}$. Furthermore, the HDL particles present marked structural, physiochemical, compositional and functional heterogeneity and have significant differences in their biological properties ${ }^{[5,10,11]}$. The major apolipoproteins of HDL are apolipoprotein A-I (ApoA-I), which constitutes approximately 70\% of HDL protein and is present on virtually all HDL particles, and ApoA-II, which constitutes approximately $20 \%$ of HDL protein and is present on about two-thirds of HDL particles in humans ${ }^{[5,12]}$.

On the other hand, the structure of the HDL particles is very complex. Mass spectrometry studies have shown that the HDL particles carry an array of proteins, which are engaged in lipid metabolism but also affect complement regulation, acute-phase response and proteinase inhibition ${ }^{[5,13]}$. Moreover, lipidomic studies have identified in excess of 200 molecular lipid species in normolipidemic HDL, including phospholipids, sphingolipids, steroids, cholesteryl esters (CEs), triglycerides, diacylglycerides, monoacylglycerides and free fatty acids ${ }^{[5,14]}$.

Given the above heterogeneity of HDL particles and their structural complexity, it becomes easily understandable that any modifications of the components of the HDL particles may alter their functionality and potentially render HDL dysfunctional.

\section{FACTORS AFFECTING HDL FUNCTIONALITY}

Certain genetic, environmental and pathophysiologic conditions can influence the HDL cardioprotective effects by disrupting its protein components, lipid content, or by promoting modifications in the enzymes responsible for HDL metabolism. 
Systemic states, such as inflammation and its equivalent acute phase response (observed after surgery and during infection or trauma), can induce significant changes on the HDL particle. During acute phase response, pro-inflammatory cytokines promote changes in the structure of plasma proteins, including those of the HDL particle. Interleukin-1 beta (IL-1 $\beta$ ), IL- 6 and tumor necrosis factor- $\alpha$ are released during acute phase response and promote the synthesis of serum amyloid A (SAA) and group IIA secretory phospholipase A2 (sPLA2-IIA), which act as pro-inflammatory molecules. SAA interacts with HDL and may result in a faster clearance of the HDL particle, resulting in reduced HDL and ApoA-I plasma levels. In addition, SAA promotes the loss of the anti-inflammatory activity of HDL and renders the HDL particle pro-inflammatory ${ }^{[15-17]}$. With regard to sPLA2-IIA, its activation promotes the breakdown of HDL phospholipids with subsequent accumulation of two proatherogenic and pro-inflammatory lipid products, lysophospholipids and fatty acids ${ }^{[18]}$, which can also disrupt HDL protein structure ${ }^{[19]}$.

Furthermore, in pro-inflammatory states, Apo-AI becomes a substrate for myeloperoxidase (MPO), a protein released by macrophages, monocytes and neutrophils, which catalyzes the chlorination or nitration of tyrosyl residues of ApoA-I molecules in HDL. MPO promotes oxidative damage of the HDL particle, which leads to a significant reduction of its anti-inflammatory properties, thus rendering HDL dysfunctional ${ }^{[20]}$.

Oxidized LDL is a powerful inducer of atherogenesis due to its role in endothelial dysfunction and foam cell formation. The mechanism by which oxidized LDL promotes atherogenesis involves the promotion of monocyte adhesion to the endothelium via activation of macrophages and mast cells ${ }^{[21]}$. As it was alluded to earlier in this review, under normal conditions, HDL has antioxidant properties and prevents oxidation of LDL, which contribute to its cardioprotective effect. However, in pro-inflammatory environments, HDL may also lose its ability to inhibit monocyte migration within the arterial wall and thus lose its antioxidative effects on LDL particles ${ }^{[22,23]}$.

Another factor that can modify the antiatherogenic properties of HDL is the alteration in the HDL lipid composition. Reorganization of HDL lipid components due to an upregulation of the activity of CE transfer protein, as observed in insulin resistance states, such as the metabolic syndrome, can modify the $\mathrm{CE} /$ triacylglyceride (TAG) ratio in HDL, which plays a crucial role for the antioxidant activity of HDL. Furthermore, increased TAG content in the lipid core may also cause dysregulation of CE transfer through scavenger receptor class B type I, therefore impairing $\mathrm{RCT}^{[24]}$.

It has been also shown that certain disease states may impair HDL function. Disorders such as atherosclerosis and type 2 diabetes mellitus promote a subclinical chronic inflammatory microenvironment at a biomolecular level, eliciting protein remodeling of HDL with subsequent disruption of its antiatherogenic, antioxidative and anti-inflammatory properties ${ }^{[2,26]}$. Furthermore, ApoA-I glycation impairs HDL functionality ${ }^{[27]}$, leading to the impairment of the anti-atherogenic ${ }^{[28]}$ and anti-inflammatory ${ }^{[29]}$ properties of HDL.

Environmental factors have also a significant impact on HDL function. Factors that alter HDL functionality include smoking, obesity and dietary habits. HDL is susceptible to oxidative modifications by cigarette smoking. As a result, HDL loses its atheroprotective properties in smokers and becomes dysfunctional ${ }^{[30]}$. With regard to obesity, there is evidence that it may reduce CEC and impair HDL functionality ${ }^{[31]}$. In addition, consumption of saturated fat has been shown to impair arterial endothelial function and reduce the anti-inflammatory activity of HDL. On the contrary, the anti-inflammatory activity of HDL is enhanced after consumption of polyunsaturated fat ${ }^{[32]}$.

\section{IMPACT OF DYSFUNCTIONAL HDL ON CVD}

There is extensive evidence from clinical studies confirming the adverse role of dysfunctional HDL on atherogenesis and the risk for CVD. 
In a post hoc analysis of two large prospective studies, the IDEAL (Incremental Decrease in End Points through Aggressive Lipid Lowering) trial and the EPIC (European Prospective Investigation into Cancer and Nutrition)-Norfolk case-control study, it was shown that very high plasma HDL-C levels ( $\geq 70 \mathrm{mg} / \mathrm{dL}$ ) and very large HDL particles $(>9.53 \mathrm{~nm}$ ) conferred an increased risk for coronary artery disease (CAD) when levels of ApoA-I and ApoB were kept constant ${ }^{[33]}$. This observation may be explained by the hypothesis that very large, cholesterol-enriched HDL particles, may at some point become cholesterol donors rather than acceptors and thus become pro-atherogenic ${ }^{[33]}$. This hypothesis was supported in another community-based cohort study, in which it was clearly shown that cholesterol-overloaded HDL particles were independently associated with progression of carotid atherosclerosis in a population free of CVD. More specifically, participants with the highest estimated number of cholesterol molecules per HDL particle ( $\geq 53.0)$ had 1.56fold increased progression of carotid atherosclerosis, as compared with those with the lowest estimated number of cholesterol molecules per HDL particle $(<41.0)^{[5,34]}$. Furthermore, in a very recent study of two large population-based cohorts in Denmark (52,268 men and 64,240 women), it was clearly shown that in men and women in the general population extremely high HDL-C levels were paradoxically associated with high all-cause mortality risk ${ }^{[35]}$. In addition, in a large-scale pooled analysis of 9 Japanese cohorts, which included 43,407 participants, it was again shown that extremely high HDL-C levels led to an increase of atherosclerotic CVD mortality ${ }^{[36]}$.

As it was mentioned earlier in this review, CEC from macrophages is currently considered an important metric of HDL function. In this regard, multiple studies have shown an inverse relationship between CEC and the incidence of cardiovascular events, independent to HDL-C levels ${ }^{[7,37,38]}$. In the Dallas heart study, a multiethnic, population-based, cohort study, in which the HDL cholesterol level, HDL particle concentration, and CEC were measured at baseline in 2,924 adults free from CVD over a mean follow-up period of 9.4 years, there was a $67 \%$ reduction in the risk for cardiovascular events in the highest quartile of CEC, as compared to the lowest quartile ${ }^{[7]}$. This again proves that dysfunctional HDL with low CEC may be an important factor in atherogenesis.

In addition, it has been shown that HDL and ApoA-I recovered from human atheroma are dysfunctional and are extensively oxidized by MPO. More specifically, while the amount of circulating ApoA-I that contains a 2-OH-Trp72 group (oxTrp72-ApoA-I) is minimal under normal conditions, it accounts for $20 \%$ of the ApoA-I in atherosclerotic arteries. Increased levels of oxTrp72-ApoA-I have been linked to an increased risk for $\mathrm{CVD}^{[39]}$. Most importantly, there is evidence showing that dysfunctional HDLs with diminished antiinflammatory activity are present in patients with CAD and they are actually found in higher abundance in patients with acute coronary syndrome (ACS) than in patients with stable angina ${ }^{[40]}$.

Furthermore, as it was alluded to earlier, HDL isolated from patients with CAD (but not HDL from healthy subjects) exhibits a pro-inflammatory rather than an anti-inflammatory effect when exposed to endothelial cells. In addition, HDL from patients with CAD (in contrast to HDL from healthy subjects) did not stimulate endothelial cell NO production, due to inhibition of the activation of endothelial NO synthase, leading to the loss of the endothelial anti-inflammatory and repair-stimulating effects of HDL in patients with $\mathrm{CAD}^{[41,42]}$.

The above were also corroborated in a large clinical study, which included 1,548 patients with CAD undergoing coronary artery bypass grafting. This study clearly demonstrated that higher pre-operative HDL-C levels were not associated with a reduction but rather with a clear tendency for an increase in the occurrence of major adverse cardiovascular events ${ }^{[43]}$. This was attributed to the presence of dysfunctional HDL in patients with CAD, as it was clearly alluded above.

\section{CONCLUSION}

From the above review of the scientific and clinical data, it becomes evident that the HDL particles possess potent cardioprotective biological functions. In addition to their effect in the facilitation of RCT, the HDL 
particles also possess cytoprotective, anti-inflammatory, antioxidant, antiaggregating and anticoagulant properties, which enhance their protective effect against CVD. These cardioprotective properties of HDL are not solely dependent on the HDL plasma concentration but also depend on HDL functionality. This was confirmed in a large meta-analysis of 108 randomized trials involving 299,310 participants at risk for CVD. In this meta-regression analysis, it was clearly shown that simply increasing the serum levels of HDL-C does not lower the risk of coronary heart disease events, coronary heart disease deaths, or total deaths ${ }^{[44]}$. On the other hand, there is extensive evidence that under certain conditions, such as the oxidative environment of the acute-phase response, the HDL particles may lose their anti-inflammatory properties and become pro-inflammatory and pro-atherogenic. Thus, future therapeutic agents targeting HDL may be required to enhance HDL functionality rather than simply increase HDL-C concentration.

\section{DECLARATIONS}

\section{Authors' contributions}

Conception and design, jointly developed the structure and arguments for the paper, made critical revisions and approved final version: Kosmas CE

Wrote the first draft of the paper: Kosmas CE, Silverio D

Contributed to the writing of the paper: Sourlas A, Montan PD, Guzman E

Data collection, analysis and interpretation, final approval of manuscript: Kosmas CE, Silverio D, Sourlas A, Montan PD, Guzman E

\section{Availability of data and materials}

Data and materials used to help write this article are available for public access.

\section{Financial support and sponsorship}

None.

\section{Conflicts of interest}

All authors declared that there are no conflicts of interest.

\section{Ethical approval and consent to participate}

Not applicable.

\section{Consent for publication}

Not applicable.

\section{Copyright}

(c) The Author(s) 2019.

\section{REFERENCES}

1. Barter P, Gotto AM, LaRosa JC, Maroni J, Szarek M, et al. HDL cholesterol, very low levels of LDL cholesterol, and cardiovascular events. N Engl J Med 2007;357:1301-10.

2. Jafri H, Alsheikh-Ali AA, Karas RH. Meta-analysis: statin therapy does not alter the association between low levels of high-density lipoprotein cholesterol and increased cardiovascular risk. Ann Intern Med 2010;153:800-8.

3. Rye KA, Barter PJ. Cardioprotective functions of HDLs. J Lipid Res 2014;55:168-79.

4. Barter PJ, Baker PW, Rye KA. Effect of high-density lipoproteins on the expression of adhesion molecules in endothelial cells. Curr Opin Lipidol 2002;13:285-8.

5. Kosmas CE, Martinez I, Sourlas A, Bouza KV, Campos FN, et al. High-density lipoprotein (HDL) functionality and its relevance to atherosclerotic cardiovascular disease. Drugs Context 2018;7:212525.

6. Favari E, Thomas MJ, Sorci-Thomas MG. High-density lipoprotein functionality as a new pharmacological target on cardiovascular disease: unifying mechanism that explains high-density lipoprotein protection toward the progression of atherosclerosis. $\mathrm{J}$ Cardiovasc Pharmacol 2018;71:325-31. 
7. Rohatgi A, Khera A, Berry JD, Givens EG, Ayers CR, et al. HDL cholesterol efflux capacity and incident cardiovascular events. N Engl J Med 2014;371:2383-93.

8. Navab M, Reddy ST, Van Lenten BJ, Fogelman AM. HDL and cardiovascular disease: atherogenic and atheroprotective mechanisms. Nat Rev Cardiol 2011;8:222-32.

9. Tailleux A, Fruchart JC. HDL heterogeneity and atherosclerosis. Crit Rev Clin Lab Sci 1996;33:163-201.

10. Kosmas CE, Christodoulidis G, Cheng JW, Vittorio TJ, Lerakis S. High-density lipoprotein functionality in coronary artery disease. Am J Med Sci 2014;347:504-8.

11. Kontush A, Lhomme M, Chapman MJ. Unraveling the complexities of the HDL lipidome. J Lipid Res 2013;54:2950-63.

12. Rader DJ. Molecular regulation of HDL metabolism and function: implications for novel therapies. J Clin Invest 2006;116:3090-100.

13. Vaisar T, Pennathur S, Green PS, Gharib SA, Hoofnagle AN, et al. Shotgun proteomics implicates protease inhibition and complement activation in the antiinflammatory properties of HDL. J Clin Invest 2007;117:746-56.

14. Kontush A, Lhomme M, Chapman MJ. Unraveling the complexities of the HDL lipidome. J Lipid Res 2013;54:2950-63

15. Tölle M, Huang T, Schuchardt M, Jankowski V, Prüfer N, et al. High-density lipoprotein loses its anti-inflammatory capacity by accumulation of pro-inflammatory-serum amyloid A. Cardiovasc Res 2012;94:154-62.

16. Jahangiri A. High-density lipoprotein and the acute phase response. Curr Opin Endocrinol Diabetes Obes 2010;17:156-60.

17. Van Lenten BJ, Hama SY, de Beer FC, Stafforini DM, McIntyre TM, et al. Anti-inflammatory HDL becomes pro-inflammatory during the acute phase response. Loss of protective effect of HDL against LDL oxidation in aortic wall cell cocultures. J Clin Invest 1995;96:2758-67.

18. Yamamoto K, Isogai Y, Sato H, Taketomi Y, Murakami M. Secreted phospholipase A2, lipoprotein hydrolysis, and atherosclerosis: integration with lipidomics. Anal Bioanal Chem 2011;400:1829-42.

19. Kar S, Patel MA, Tripathy RK, Bajaj P, Suvarnakar UV, et al. Oxidized phospholipid content destabilizes the structure of reconstituted high density lipoprotein particles and changes their function. Biochim Biophys Acta 2012;1821:1200-10.

20. Kameda T, Ohkawa R, Yano K, Usami Y, Miyazaki A, et al. Effects of myeloperoxidase-induced oxidation on antiatherogenic functions of high-density lipoprotein. J Lipids 2015;2015:592594.

21. Chen C, Khismatullin DB. Oxidized low-density lipoprotein contributes to atherogenesis via co-activation of macrophages and mast cells. PLoS One 2015;10:e123088.

22. Otocka-Kmiecik A, Mikhailidis DP, Nicholls SJ, Davidson M, Rysz J, et al. Dysfunctional HDL: a novel important diagnostic and therapeutic target in cardiovascular disease? Prog Lipid Res 2012;51:314-24.

23. Navab M, Hama SY, Hough GP, Subbanagounder G, Reddy ST, et al. A cell-free assay for detecting HDL that is dysfunctional in preventing the formation of or inactivating oxidized phospholipids. J Lipid Res 2001;42:1308-17.

24. Salazar J, Olivar LC, Ramos E, Chávez-Castillo M, Rojas J, et al. Dysfunctional high-density lipoprotein: an innovative target for proteomics and lipidomics. Cholesterol 2015;2015:296417.

25. Chait A, Han CY, Oram JF, Heinecke JW. Thematic review series: the immune system and atherogenesis. Lipoprotein-associated inflammatory proteins: markers or mediators of cardiovascular disease? J Lipid Res 2005;46:389-403.

26. Nobécourt E, Jacqueminet S, Hansel B, Chantepie S, Grimaldi A, et al. Defective antioxidative activity of small dense HDL3 particles in type 2 diabetes: relationship to elevated oxidative stress and hyperglycaemia. Diabetologia 2005;48:529-38.

27. Hedrick CC, Thorpe SR, Fu MX, Harper CM, Yoo J, et al. Glycation impairs high-density lipoprotein function. Diabetologia 2000;43:312-20.

28. Hoang A, Murphy AJ, Coughlan MT, Thomas MC, Forbes JM, et al. Advanced glycation of apolipoprotein A-I impairs its antiatherogenic properties. Diabetologia 2007;50:1770-9.

29. Nobécourt E, Tabet F, Lambert G, Puranik R, Bao S, et al. Nonenzymatic glycation impairs the antiinflammatory properties of apolipoprotein A-I. Arterioscler Thromb Vasc Biol 2010;30:766-72.

30. He BM, Zhao SP, Peng ZY. Effects of cigarette smoking on HDL quantity and function: implications for atherosclerosis. J Cell Biochem 2013;114:2431-6.

31. McMorrow AM, O'Reilly M, Connaughton RM, Carolan E, O'Shea D, et al. Obesity and dietary fat modulate HDL function in adolescents: results from a cross-sectional analysis and a randomized, placebo-controlled, crossover trial. FASEB J 2016;30:130.

32. Nicholls SJ, Lundman P, Harmer JA, Cutri B, Griffiths KA, et al. Consumption of saturated fat impairs the anti-inflammatory properties of high-density lipoproteins and endothelial function. J Am Coll Cardiol 2006;48:715-20.

33. van der Steeg WA, Holme I, Boekholdt SM, Larsen ML, Lindahl C, et al. High-density lipoprotein cholesterol, high-density lipoprotein particle size, and apolipoprotein A-I: significance for cardiovascular risk: the IDEAL and EPIC-Norfolk studies. J Am Coll Cardiol 2008;51:634-42.

34. Qi Y, Fan J, Liu J, Wang W, Wang M, et al. Cholesterol-overloaded HDL particles are independently associated with progression of carotid atherosclerosis in a cardiovascular disease-free population: a community-based cohort study. J Am Coll Cardiol 2015;65:355-63.

35. Madsen CM, Varbo A, Nordestgaard BG. Extreme high high-density lipoprotein cholesterol is paradoxically associated with high mortality in men and women: two prospective cohort studies. Eur Heart J 2017;38:2478-86.

36. Hirata A, Sugiyama D, Watanabe M, Tamakoshi A, Iso H, et al. Association of extremely high levels of high-density lipoprotein cholesterol with cardiovascular mortality in a pooled analysis of 9 cohort studies including 43,407 individuals: the EPOCH-JAPAN study. J Clin Lipidol 2018;12:674-84.

37. Khera AV, Cuchel M, de la Llera-Moya M, Rodrigues A, Burke MF, et al. Cholesterol efflux capacity, high-density lipoprotein function, 
and atherosclerosis. N Engl J Med 2011;364:127-35.

38. Ogura M, Hori M, Harada-Shiba M. Association between cholesterol efflux capacity and atherosclerotic cardiovascular disease in patients with familial hypercholesterolemia. Arterioscler Thromb Vasc Biol 2016;36:181-8.

39. Huang Y, DiDonato JA, Levison BS, Schmitt D, Li L, et al. An abundant dysfunctional apolipoprotein A1 in human atheroma. Nat Med 2014;20:193-203.

40. Carnuta MG, Stancu CS, Toma L, Sanda GM, Niculescu LS, et al. Dysfunctional high-density lipoproteins have distinct composition, diminished anti-inflammatory potential and discriminate acute coronary syndrome from stable coronary artery disease patients. Sci Rep 2017;7:7295.

41. Kratzer A, Giral H, Landmesser U. High-density lipoproteins as modulators of endothelial cell functions: alterations in patients with coronary artery disease. Cardiovasc Res 2014;103:350-61.

42. Besler C, Heinrich K, Rohrer L, Doerries C, Riwanto M, et al. Mechanisms underlying adverse effects of HDL on eNOS-activating pathways in patients with coronary artery disease. J Clin Invest 2011;121:2693-708.

43. Angeloni E, Paneni F, Landmesser U, Benedetto U, Melina G, et al. Lack of protective role of HDL-C in patients with coronary artery disease undergoing elective coronary artery bypass grafting. Eur Heart J 2013;34:3557-62.

44. Briel M, Ferreira-Gonzalez I, You JJ, Karanicolas PJ, Akl EA, et al. Association between change in high density lipoprotein cholesterol and cardiovascular disease morbidity and mortality: systematic review and meta-regression analysis. BMJ 2009;338:b92. 